Bio - grafia. Escritos sobre la Biología y su Enseñanza. ISSN 2027

Edición Extraordinaria. p.p. 1447-1459

Memorias del VIII Encuentro Nacional de Experiencias en Enseñanza de la Biología y la Educación Ambiental. III Congreso Nacional de Investigación en Enseñanza de la Biología.

\title{
LA ENSEÑANZA DE LA FAUNA EN EDUCACION INICIAL, UNA PROPUESTA ALTERNATIVA ${ }^{1}$
}

\author{
WILDLIFE EDUCATION INITIAL EDUCATION, AN ALTERNATIVE PROPOSAL
}

B

I

0

$P$

0

$\mathrm{N}$

$E$

$\mathrm{N}$

C

I

A (rete the teaching of science way.

Palabras claves: Ciencias, medio ambiente, fauna, género, y educación inicial.

\footnotetext{
${ }^{1}$ ANACONA MARÍA OMAIRA; TRUJILLO SILVA MARY LUZ; NAVARRO GONZÁLEZ ADRIANA Colegio Gabriel García Márquez (Las Violetas) IED. Profesoras de Primera Infancia. Secretaria de Educación Distrital. Correos: anaconaupn@yahoo.com; osmimary@hotmail.com; adripreescolar@hotmail.com
} 
Bio - grafia. Escritos sobre la Biología y su Enseñanza. ISSN 2027

Edición Extraordinaria. p.p. 1447-1459

Memorias del VIII Encuentro Nacional de Experiencias en Enseñanza de la Biología y la Educación Ambiental. III Congreso Nacional de Investigación en Enseñanza de la Biología.

\section{Introducción}

Romper con el imaginario de la ciencia moderna occidental con la perspectiva disciplinar y cruzar las fronteras hacia las ciencias más humanas, sociales y culturalmente pertinentes al contexto partículas de la escuela son desafíos que los educadores deben asumir para corresponder a una enseñanza y aprendizaje en condiciones de igualdad en donde el conocimiento científico no sea el objetivo tradicional de interés sino que el conocimiento tenga la posibilidad de ser reconocido en sus diferentes actores "la educación es un campo de investigación actual que reconoce un gran número de interacciones posibles entre profesores y estudiantes" (Lee, 2013), en ello la escuela juega un papel trascendental para la enseñanza de las ciencias, siendo un campo que permite la interrelación cultural y posibilita el diálogo entre sus diversos actores como los estudiantes, profesores, científicos, políticos, en si en toda la comunidad educativa, teniendo en cuenta los cambios importantes que están sucediendo en el entorno escolar a partir de las dinámicas locales, distritales, nacionales y en general globales. En este orden, es importante destacar las actitudes de los estudiantes hacia las ciencias, en donde distintos estudios han demostrado que se presentan cambios en el proceso escolar (Murphy y Beggs, citado por Molina, Carriazo y Casas, 2013).

Por otro lado, la identificación de las actitudes que tienen los niños y niñas en la educación inicial puede contribuir hacia la estructuración de propuestas en ciencias que sean pertinentes a sus intereses y así mismo aporten en la construcción del conocimiento. En este sentido, es importante identificar estas actitudes hacia las ciencias desde la perspectiva de género, al respecto, algunos estudios evidencian actitudes desfavorables de las mujeres hacia las ciencias, (George, citado por Molina, Carriazo y Casas, 2013) posiblemente por causa del ambiente escolar e influencia por parte del profesorado (Mirandes, Sanmartí y Tarín, citado por Molina, Carriazo y Casas, 2013).

También es primordial mirar el perfil del profesorado desde su formación profesional, se han realizado estudios sobre las creencias de los profesores de preescolar y primaria acerca de la ciencia y en particular que los profesores que realizan su labor en contextos rurales que tienen una mirada de la ciencia reduccionista hacia el método científico (Ruíz, Martínez y Parga, 2009) lo que conlleva a considerar de importancia el estudio de las creencias de los profesores, porque estas orientan las acciones del profesor en el proceso de enseñanza, en donde se percibe que es importante desarrollar proceso de lectura, escritura y el desarrollo de las matemáticas, dejando a un lado las ciencias y finalmente se va aumentando el desinterés hacia las ciencias a medida que los estudiantes avanzan en sus 
Bio - grafia. Escritos sobre la Biología y su Enseñanza. ISSN 2027

Edición Extraordinaria. p.p. 1447-1459

Memorias del VIII Encuentro Nacional de Experiencias en Enseñanza de la Biología y la Educación Ambiental. III Congreso Nacional de Investigación en Enseñanza de la Biología.

respectivos grados (Pell y Jarvis, citado por Molina, Carriazo y Casas, 2013). Además, los profesores consideran que no es importante la formación didáctica y pedagógica, que tan solo saber la materia es suficiente para la enseñanza, además se evidencia que los conceptos movilizados en el aula no están en relación significativa para el estudiante (García et al, citado por Ruíz, Martínez y Parga, 2009).

La perspectiva de medio ambiente

La relación del hombre con el medio ambiente y en particular con la fauna hace que se establezcan vínculos de preservación, conservación, concientización y en general de cuidado y respeto a la naturaleza. La especie humana debe ser consciente de su interacción con el medio ambiente. Por lo tanto, es importante indagar que tan responsable somos con el contexto que habitamos en particular en aquellos escenarios que siendo rurales y que tienen gran riqueza ambiental, se están urbanizando principalmente porque no hay terrenos para construcción las periferias de la ciudad están teniendo construcciones que representan interferencia con el medio ambiente natural propio del sector. Por consiguiente la escuela representa un vínculo importante para sensibilizar a la comunidad con su entorno ambiental.

La biodiversidad es la mayor riqueza que tiene un país, y en este sentido, es obligación que desde la escuela y en particular desde la Educación Inicial se sensibilice sobre el cuidado y protección de fauna, flora, fuentes hídricas y en general de todos los recursos naturales (renovables y no renovables) y dejar de percibir el medio ambiente como proveedor inagotable.

La fauna es uno de los componentes principales de la biodiversidad. Los animales han establecido relaciones directas con las personas, están relaciones han sido emocionales, económicas, medicinales, y otras propias de cada cultura Páramo y Galvis (2010, p 111) realizaron un estudio para "identificar los vínculos que desarrollan niños de diferentes contextos culturales y regionales de Colombia (de la sociedad mayoritaria e Indígena) sobre los animales a través de sus constructos personales".

En la actualidad los diferentes movimientos sociales y ambientales que defienden el cuidado de la biodiversidad y el medio ambiente muestran intereses de estudio entre las actitudes de las personas hacia la fauna (Kendll y Lobao, citado por Galvis y Páramo, 2010) y flora, además de movimientos para proteger el agua y el aire.

En los últimos años es evidente que la crisis ambiental está siendo discutida en distintos escenarios, en uno de ellos es la escuela lugar que debe generar espacio de reflexión, 
Bio - grafia. Escritos sobre la Biología y su Enseñanza. ISSN 2027

Edición Extraordinaria. p.p. 1447-1459

Memorias del VIII Encuentro Nacional de Experiencias en Enseñanza de la Biología y la Educación Ambiental. III Congreso Nacional de Investigación en Enseñanza de la Biología.

discusión y debate para formar en cultura del cuidado del medio ambiente y así en un futuro prever problemáticas como medio ambiente.

En este sentido, Milano (2010) reconoce que es necesario promover desde la escuela una educación ambiental a partir de la enseñanza de las ciencias con procesos dinámicos, didácticos y pedagógicos que generen conciencia de las problemáticas ambientales de la comunidad que pueden efectos adversos tanto en lo local, nacional e internacional

La educación de las ciencias debe ser desde un enfoque multidisciplinar que permita configurar desde diversas perspectivas conceptualizaciones e idealización tan válidas como las de la ciencia occidental.

Lineamientos curriculares en la Educación Inicial

Milano,(2014) expresa la necesidad de profundizar el conocimiento y análisis de la política educativa vigente, lo que implica pensar en las formas de organizar y avaluar el conocimiento, con la intervención de políticas educativas que puedan favorecer e impactar en el escenario educativo en particular es exigido por la misma institución.

La institución escolar es aquella estructura social que tiene como función garantizar la apropiación de saberes considerados culturalmente válidos y socialmente relevantes "Sabemos que la escuela es un instrumento de inclusión social, cuando, además de garantizar el acceso a todo la población, logra formar en los conocimientos y los valores que la sociedad de hoy y el futuro demandan: inclusión con aprendizaje" (Misirlis, citado por Milano, 2014).

Por consiguiente, se debe proponer alternativas de enseñanza que tenga en cuenta los conocimientos de los niños para educación contextual que contribuya con otras formas de interactuar con las ciencias para replantear el currículo con el aporte de la comunidad educativa y que tengan relación con los Lineamientos Pedagógicos y Curriculares para la Educación Inicial.

"De esta manera, al ser el juego, la literatura, las diversas expresiones artísticas y la exploración del medio, las actividades propias que caracterizan a la primera infancia, el objetivo de la educación inicial no es enseñar contenidos temáticos de la manera en que se hace en la básica primaria, o tratar al niño como alumno en situación escolar, sino desplegar diferentes oportunidades para potenciar su desarrollo" 
Bio - grafia. Escritos sobre la Biología y su Enseñanza. ISSN 2027

Edición Extraordinaria. p.p. 1447-1459

Memorias del VIII Encuentro Nacional de Experiencias en Enseñanza de la Biología y la Educación Ambiental. III Congreso Nacional de Investigación en Enseñanza de la Biología.

(MEN, 2014. p 42)

El aprendizaje se fortalece desde los intereses de los niños y las niñas partiendo de los conocimientos de su vida cotidiana. En esta etapa se les tiene en cuenta sus preguntas e intereses, reconociendo el ser y el estar en el mundo de la exploración, jugar, interactuar consigo mismo, inventar, crear, imaginar y construir, reconociendo sus fortalezas y habilidades de los niños y las niñas en la cual se implementan procesos de desarrollo de aprendizaje significativo, formándolos como sujetos de derechos y deberes para actuar en una sociedad cambiante. (MEN, 2014. p 43).

En relación con la formación de profesores en ciencias, Obregoso, Vallejo y Valbuena, (2013) afirman que hay una auto formación en las ciencias por interés particular de los profesores porque las dinámicas de la escuela hacen que los profesores en su mayoría deban enseñar todas las áreas, pero las motivaciones personales hacen que haya una tendencia a determinada área para el caso particular corresponde a las ciencias, sin embargo, también esta lo contrario y es enseñar las ciencias por obligación al currículo, siendo las ciencias un medio que posibilita el desarrollar de las habilidades comunicativas, cognitivas y actitudinales que en su conjunto es la formación ciudadana que la escuela debe proyectar para una sociedad responsable con el medio ambiente.

Metodología

Se realiza un estudio de carácter descriptivo exploratorio y se centra principalmente en estudiar las concepciones que tiene tres grupos de niños y niñas de Pre-Jardín y Jardín en la Educación Inicial, para ello se utilizarán diferentes instrumentos como encuestas, grabaciones de audio y videos y fotografías.

\section{Participantes}

La comunidad educativa está ubicada en la localidad quinta, (Usme), en el Colegio Gabriel García Márquez (Las Violetas IED), del barrio Villa Diana, con una población de 1.450 estudiantes desde Educación Inicial hasta $11^{\circ}$ grado, en dos jornadas en una sede, y 70 educadores.

La experiencia investigativa cuenta con la participación de 3 profesoras de educación inicial licenciadas en preescolar y psicopedagogía (2 profesoras de Pre-jardín y 1 de jardín) y 36 estudiantes de Pre-jardín y 20 estudiantes de Jardín con edades entre tres y cuatro años, quienes participan del programa de Educación Inicial, en jornada única. 
Bio - grafia. Escritos sobre la Biología y su Enseñanza. ISSN 2027

Edición Extraordinaria. p.p. 1447-1459

Memorias del VIII Encuentro Nacional de Experiencias en Enseñanza de la Biología y la Educación Ambiental. III Congreso Nacional de Investigación en Enseñanza de la Biología.

\section{Instrumentos}

El primer instrumento "RECONOCIENDO LOS ANIMALES DE MI CONTEXTO" se aplicó a los niños y niñas de Educación Inicial con la intención de identificar sus conocimientos cotidianos sobre su contexto y en particular sobre la fauna, además de su relación con el medio ambiente y las ciencias.

Las preguntas fueron validadas previamente por dos profesores de ciencias externos a la investigación quienes hicieron las respectivas observaciones al instrumento.

Procedimiento

La información sobre su contexto y en particular sobre la fauna y su relación con el medio ambiente fue recogida mediante una encuesta individual, la cual fue grabada debido a que los niños no tienen proceso de escritura, sin embargo tienen un desarrollo de oralidad lo cual permitió ir grabando y transcribiendo la encuesta, posteriormente se sistematizo y se hizo el respectivo análisis para proceder a identificar los conocimientos que tienen los niños y niñas de la fauna propia del territorio y relacionarla con investigaciones de fauna desde el ámbito académico.

Resultados parciales

En la encuesta "Reconociendo los animales de mi contexto" aplicada a una muestra de 30 estudiantes (15 niñas y 15 niños) de pre-escolar y jardín se hallaron las siguientes concepciones sobre la fauna de su contexto.

Para los niños y las niñas los animales más representativos de su contexto es la vaca, seguidamente los perros y los gatos, puede ser que tienen como mascota y los que habitan en la calle, el conejo es otro de los animales que reconocen. El pájaro es poco reconocido a pesar de estar en una zona de bosque, en relación con el león no es un animal propio de esta zona y tal vez los niños lo relacionan con algún juguete o peluche o programa de televisión (Ver tabla 1) 
Bio - grafia. Escritos sobre la Biología y su Enseñanza. ISSN 2027

Edición Extraordinaria. p.p. 1447-1459

Memorias del VIII Encuentro Nacional de Experiencias en Enseñanza de la Biología y la Educación Ambiental. III Congreso Nacional de Investigación en Enseñanza de la Biología.

Tabla 1. Animales comunes para niños y niñas

\begin{tabular}{|l|c|c|}
\hline Animal & Niñas & Niños \\
\hline Vaca & 14 & 13 \\
\hline perro & 9 & 9 \\
\hline Gato & 6 & 3 \\
\hline conejo & 2 & 3 \\
\hline leones & 1 & 2 \\
\hline Pájaro & 1 & 2 \\
\hline
\end{tabular}

Las niñas nombran el oso y el águila a pesar de no ser un animal propio de nuestro contexto, tal vez lo relacionan con un juguete o con historias de cuentos e imágenes, las mariposas y los ratones si habitan el contexto (Ver tabla 2).

Tabla 2. Animales comunes únicamente para las niñas

\begin{tabular}{|l|c|}
\hline Animal & $\begin{array}{l}\text { Veces } \\
\text { nombrado }\end{array}$ \\
\hline Osos & 2 \\
\hline Águila & 1 \\
\hline Mariposa & 1 \\
\hline Ratones & 1 \\
\hline
\end{tabular}

Los niños nombran 10 animales que no son comunes para las niñas, destacan el pollo y el pato que son propios de este contexto, también destacan las palomas, gallinas, arañas, cucarachas, serpientes y gusanos que también son propias del contexto en particular las cucarachas pueden significar problemas de higiene. Los gusanos son propios del contexto semi-rural, en cambio se identifica una descontextualización con el pulpo al parecer influyo 
Edición Extraordinaria. p.p. 1447-1459

Memorias del VIII Encuentro Nacional de Experiencias en Enseñanza de la Biología y la Educación Ambiental. III Congreso Nacional de Investigación en Enseñanza de la Biología.

una de las actividades realizadas por las docentes de compensar relacionada con el Mar, esto evidencia que no hay una relación del contexto real con el currículo (Ver tabla 3)

Tabla 3. Animales comunes únicamente para los niños

\begin{tabular}{|l|c|}
\hline Animal & $\begin{array}{l}\text { Veces } \\
\text { nombrado }\end{array}$ \\
\hline Pollo & 3 \\
\hline Pato & 2 \\
\hline Paloma & 1 \\
\hline Gallina & 1 \\
\hline Pulpo & 1 \\
\hline Elefante & 1 \\
\hline Araña & 1 \\
\hline Cucaracha & 1 \\
\hline Serpiente & 1 \\
\hline Gusano & 1 \\
\hline
\end{tabular}

En general es importante destacar que los niños destacan más animales diferentes en su hábitat en comparación con los cuatro animales que nombran las niñas.

También son evidentes que nombran animales que tal vez son de juguete y no son del contexto, esto evidencia el maravilloso mundo de las niñas y los niños para ir auscultando para poder estableciendo relaciones con una enseñanza de las ciencias contextualizada como posibilitadora para la construcción de conocimiento.

De los animales en general las niñas y niños destacan más la vaca en cualidades como un animal que proporciona leche y carne teniendo una visión consumista, por otro lado destacan el perro como mascota y que genera afecto y compañía. 
Bio - grafia. Escritos sobre la Biología y su Enseñanza. ISSN 2027

Edición Extraordinaria. p.p. 1447-1459

Memorias del VIII Encuentro Nacional de Experiencias en Enseñanza de la Biología y la Educación Ambiental. III Congreso Nacional de Investigación en Enseñanza de la Biología.

En particular, las niñas destacan el perro como mascota y que genera afecto y compañía... Y también consideran los ratones como tiernos, a pesar de generar problemáticas de salubridad, las niñas no reconocen ello.

Los niños destacan pollos, patos, las cucarachas, las arañas y las serpientes. En relación con las cucarachas, los niños no la consideran como un insecto que es identificador de problemas de higiene y al contrario se divierten al verla saltar, esto evidencia al respeto a la fauna. Con las arañas se refleja que están en sus casas y hay una relación directa (Ver tabla 4).

Tabla 4. Animales que les gustan a los niños y niñas

\begin{tabular}{|c|c|c|}
\hline & NIÑAS & NIÑOS \\
\hline perro & $\begin{array}{l}\text { Me gusta con ellos } \\
\text { Porque son pequeños }\end{array}$ & $\begin{array}{l}\text { Porque tenemos uno en casa } \\
\text { Porque tiene a su perra luna y ella } \\
\text { juega }\end{array}$ \\
\hline Gatos & $\begin{array}{l}\text { Me gusta jugar con ellos } \\
\text { Porque es tierno } \\
\text { Porque tengo un gato que se llama yepa } \\
\text { Porque si me gusta }\end{array}$ & \\
\hline vaca & $\begin{array}{l}\text { Porque son bonitas } \\
\text { y porque comen pasto } \\
\text { saca leche } \\
\text { porque me gusta la leche } \\
\text { porque es tierna } \\
\text { las vacas corren y no se dejan coger } \\
\text { las vacas porque mi mamá me aman }\end{array}$ & $\begin{array}{l}\text { Porque si } \\
\text { Porque mi mamá me dijo } \\
\text { Porque me montan en la vaca } \\
\text { Porque nos da leche y carne } \\
\text { La vaca porque sale la leche } \\
\text { Vaca porque hace muuuuu } \\
\text { Porque las ves a veces } \\
\text { Porque las granjero las ordeñan }\end{array}$ \\
\hline
\end{tabular}


Bio - grafia. Escritos sobre la Biología y su Enseñanza. ISSN 2027

Edición Extraordinaria. p.p. 1447-1459

Memorias del VIII Encuentro Nacional de Experiencias en Enseñanza de la Biología y la Educación Ambiental. III Congreso Nacional de Investigación en Enseñanza de la Biología.

\begin{tabular}{|l|l|l|}
\hline & & Porque comen pasto \\
\hline ratones & Porque son tiernos & Porque si \\
\hline pollo & Patos & Porque se meten en el agua \\
\hline cucarachas & Porque saltan y las ve todos \\
\hline arañas & $\begin{array}{l}\text { Porque las ves en la casa todo el } \\
\text { tiempo }\end{array}$ \\
\hline
\end{tabular}

En general los niños justifican y nombran el gusto por 7 animales y en relación con las niñas justifican el por qué les gustan 4 animales.

Establecen una relación de interacción, faltaría aportar un consolidado dialogo sobre cuáles serían los beneficios y perjuicios o perjuicios en relación hombre - medio ambiente en particular con la fauna (Ver tabla 5).

Tabla 5. Animales que no les gustan a los niños y niñas

\begin{tabular}{|c|c|c|}
\hline ANIMAL & NIÑAS & NIÑOS \\
\hline Pollito & $\begin{array}{l}\text { Porque no } \\
\text { Porque le pica }\end{array}$ & \\
\hline Bichos & $\begin{array}{l}\text { Que caminan arrastrándose por la } \\
\text { tierra }\end{array}$ & \\
\hline Águila & Porque en la casa no hay gatos & \\
\hline Patos & Porque no & \\
\hline Tigres & Porque son de este color amarrillo & $\begin{array}{l}\text { Porque te dan miedo } \\
\text { Porque no } \\
\text { Porque come pasto }\end{array}$ \\
\hline
\end{tabular}


Bio - grafia. Escritos sobre la Biología y su Enseñanza. ISSN 2027

Edición Extraordinaria. p.p. 1447-1459

Memorias del VIII Encuentro Nacional de Experiencias en Enseñanza de la Biología y la Educación Ambiental. III Congreso Nacional de Investigación en Enseñanza de la Biología.

\begin{tabular}{|l|l|l|}
\hline Caballo & Porque no da leche y no mas & \\
\hline Perro & $\begin{array}{l}\text { Muerden a los niños y a la gente } \\
\text { Porque te mordió porque tenía } \\
\text { hambre } \\
\text { Porque hacen popo y chichi y } \\
\text { ensucian todo }\end{array}$ & Porque no me dejan dormir \\
\hline Gatos & Porque aruñan la cara de los niños & Porque son pequeñas \\
\hline serpientes & & Porque no \\
\hline Elefante & & Porque toman pasto \\
\hline Gusanos & & $\begin{array}{l}\text { Porque si } \\
\text { suben a la cabeza del niño }\end{array}$ \\
\hline Palomas & &
\end{tabular}

Para los niños y las niñas no les gustan los perros y los tigres, las niñas afirman que los perros muerden a la gente y además ensucian las calles con heces fecales y los niños expresan que no los dejan dormir, en relación con el tigre es un animal que no es propio del contexto y sin embargo genera temor.

Con las niñas es interesante como consideran los bichos que hacen que sea parte de estudio del medio ambiente. 
Bio - grafia. Escritos sobre la Biología y su Enseñanza. ISSN 2027

Edición Extraordinaria. p.p. 1447-1459

Memorias del VIII Encuentro Nacional de Experiencias en Enseñanza de la Biología y la Educación Ambiental. III Congreso Nacional de Investigación en Enseñanza de la Biología.

\section{Bibliografía}

> Alcaldía Mayor de Bogotá. (2010). Lineamiento Pedagógico y Curricular para la Educación Inicial en el Distrito. Bogotá.

Lee, O. (2013). Promoviendo la investigación científica con estudiantes de grado elemental de diversas culturas y lenguas. En: Revista Magis. 6 (12), 199-204.

> Ministerio de Educación Nacional. (2014). Serie de orientaciones pedagógicas para la educación inicial en el marco de la atención integral. Sentido de la educación inicial. Bogotá.

> Milano, E. (2010), Educar, habitar, convivir... Un Proyecto de Educación Humana y Ambiental. En: Revista Magisterio, 47, 44-47.

> Milano, E. (2014), La inclusión escolar, el niño y su derecho de aprender. En: Revista Internacional Magisterio, Nov-dic, 82- 87.

> Molina, M, Carriazo J y Casas, J. (2013). Estudio transversal de las actitudes hacia la ciencia en estudiantes de grados quinto a undécimo. Adaptación y aplicación de un instrumento para valorar actitudes. En: Revista Tecné, Episteme Didaxis TED, 33, 103-122.

> Paramo P, y Galvis C. (2010) Conceptualizaciones acerca de los animales en niños de la sociedad mayoritaria y de la comunidad indígena uitoto en Colombia. En revista Folios, 32, 111- 124.

Duíz,D., Martínez, L y Parga, D. (2009). Creencias de los profesores de Preescolar y Primaria sobre ciencia, tecnología y sociedad en el contexto de una institución escolar. En: Revista Tecné, Episteme Didaxis TED, 25, 41-61. 
Bio - grafia. Escritos sobre la Biología y su Enseñanza. ISSN 2027

Edición Extraordinaria. p.p. 1447-1459

Memorias del VIII Encuentro Nacional de Experiencias en Enseñanza de la Biología y la Educación Ambiental. III Congreso Nacional de Investigación en Enseñanza de la Biología.

> Obregoso, Y., Vallejo, y Valbuena, E. (2013). El conocimiento didáctico del contenido de las ciencias naturales en docentes en formación inicial de primaria. Un estudio de caso. En, Martínez y Valbuena. (Comp), Conocimiento Profesional del profesor de ciencias de primaria y conocimiento escolar (p 81-111). Bogotá: Universidad Distrital Francisco José de Caldas 
